\title{
Comparison Between Two Enrichment Broths for Detection of Salmonella from Reptilian Feces and Cloacal Swabs
}

\author{
Naqa Saleh Mahdi Tamimi ${ }^{1 *}$, Amir Rostami $^{2}$ and Hossein Esmaeili ${ }^{3}$ \\ ${ }^{1}$ Department of Internal and preventive Medicine; College of veterinary medicine, \\ University of Wasit. Kut, Iraq \\ ${ }^{2}$ Department of Internal Medicine; Faculty of Veterinary Medicine; University of \\ Tehran, Tehran, Iran \\ ${ }^{3}$ Department of Microbiology; Faculty of Veterinary Medicine; University of Tehran, \\ Tehran, Iran \\ *Corresponding Author: Naqa Saleh Mahdi Tamimi, Department of Internal and \\ preventive Medicine; College of veterinary medicine, University of Wasit. Kut, Iraq. \\ E-mail: naqavet2018@gmail.com,nmahdi@uowasit.edu.iq
}

Received: July 28, 2021

Published: August 31, 2021

(C) All rights are reserved by Naqa Saleh

Mahdi Tamimi., et al.

\begin{abstract}
Reptiles are shown to harbor and shed Salmonella without having any sign of illness and with notable rates that could pose a health hazard for their owners. In order to find a suitable enrichment broth for the recovery of Salmonella from these animals' feces Rappaport Vassiliadis (RV) broth was compared with selenite F (SF). Two sets of samples were collected from 270 reptiles in Tehran province which belonged to 46 reptilian species of 22 families. The specimens included 171 cloacal swabs, 20 feces, and 79 terrarium water specimens (in which the reptile defecates) according to their availability and reptile species. Each sample was separately enriched in RV and SF enrichment broths and then inoculated in three culture media to increase the possibility of Salmonella detection (CHROMagar Salmonella, MacConkey agar, and xylosine-lysin desoxycholate) and later checked for their biochemical properties as Salmonella spp. A total of 147 (54.4\%) specimens were found positive for Salmonella using both enrichment broths. The positivity rate for RV (49.6\%) was significantly higher than SF (40.4\%) showing that RV could be considered a better enrichment broth for Salmonella recovery in feces or cloacal swabs $(\mathrm{p}=0.031)$. In addition, the reptilian feces samples were more likely to have positive culture results than cloacal swabs and terrarium water in RV and SF broths ( $p=0.001$ and 0.000 , respectively); suggesting that, feces can be better specimens for detection of Salmonella intestinal shedding in reptiles or other animals.
\end{abstract}

Keywords: Enrichment Broth; Reptile; Salmonella

\section{Introduction}

Salmonellosis is an important public health problem that affects more animals and people compared to other single diseases $[1,2]$. It is a zoonotic disease and many animals act as reservoirs. Reptiles are shown to harbor Salmonella without having any sign of illness with a prevalence that could reach 90\% [3]. More than 1300 serotypes have been reported in reptiles; from which very few were isolated from human or have been associated with reptile contact and zoonosis [4]. Cases of reptile-associated salmonellosis in human were not uncommon, some of them being fatal $[5,6]$. In response to those cases, the U.S. Food and Drug Administration (FDA) banned the importation of small turtles (carapace smaller than four inches) which lead to a reduction in turtle associated salmonellosis [7].

These facts highlight the hazard that the Salmonella sp. Shed by reptiles represents for human health. It also underlines the importance of this pathogen's detection concerning health control programs [8]. Using an effective method for Salmonella detection in control programs, helps a more reliable infection rate estimation possible since using different culture methods for detection of Salmonella species has also been found to have a significant impact in the possibility of Salmonella detection [9]. When considering culture methods, selecting the appropriate enrichment broth 
is very important; especially in the feces or cloacal swabs which needs a strong inhibition of the competitive bacteria while it allows the growth of Salmonella sp. [10,11]. Therefore, this study was designed to find out which one of the two enrichment broths (Rappaport Vassiliadis or Selenite F) works better for the detection of Salmonella species in the feces and cloacal swabs of reptiles.

\section{Materials and Methods}

Sampling

A total of 270 samples from reptiles of all ages referred to the specialized veterinary clinics in Tehran were studied. Three types of samples were collected: 1-cloacal swabs (two for each reptile), 2 -feces $(10 \mathrm{~g})$, and 3 -terrarium water $(10 \mathrm{ml})$. The choice of the sample type was related to the reptilian species and the availability of the specimen. In the case of terrarium water owners were asked to keep the water unchanged for at least five days before the sample was collected in order to increase the chance of Salmonella detection. In case of unavailability of feces (even after motivation) cloacal swab was obtained.

\section{Salmonella isolation}

All samples were divided into two parts and inoculated in two enrichment broths: Rappaport Vassiliadis broth (RV) and selenite F broth (SF) (Merck, Germany). Inoculated broths were then incubated for $12-18 \mathrm{~h}$ at $42^{\circ} \mathrm{C}$ and $37^{\circ} \mathrm{C}$, respectively. Then both enrichment broths were inoculated and streaked for isolation on CHROMagar Salmonella (France), MacConkey agar (Merck), and xylosine-lysin desoxycholate (XLD) (Merck, Germany), and incubated in $37^{\circ} \mathrm{C}$ for 24 - 36h. Presumptive Salmonella colonies of each culture media were picked and subjected to biochemical tests with the use of standard media (Urea (Diffco, USA), Triple Sugar Iron (TSI) (Merck, Germany), peptone water (Difco, USA), Simmons' Citrate agar (Merck, Germany), and MRVP (Difco, USA) and procedures to make sure they belonged to the Salmonella spp.

\section{Statistical analysis}

Chi-square test was used to detect significant difference between the enrichment broths and positive Salmonella cultures. This test was also used to find out if the proportion of positive culture results in each broth was affected by the type of specimen used. In order to find out which type of specimen led to more positive culture results in each enrichment broth, multiple comparison with Bonferroni correction was conducted. The SPSS software (version
20; SPSS Inc. Chicago, IL, USA) was used for the analysis and a $\mathrm{p}$ value of less than 0.05 was considered statistically significant.

\section{Results and Discussion}

Samples culture results

Specimens including water (79 samples), feces (20 samples) and cloacal swabs (171 samples) were collected from 46 reptile species of 22 families. Of the total 270 reptiles from which samples were obtained, 82 were kept in humid, 86 in arid, and the rest, 102 were kept in semi-aquatic environments. Table 1 shows the positive and negative culture results of each enrichment broth. A total of 147 (54.4\%) specimens were found positive for Salmonella using both enrichment broths. Table 2 gives the detailed results of Salmonella cultures according to the type of the specimen used in this study.

Table 1: Distribution of Salmonella culture results of 270 reptile samples in RV and SF broths.

\begin{tabular}{|c|c|c|c|}
\hline \multirow{2}{*}{ Enrichment broth } & \multicolumn{2}{|c|}{ Culture Results } & \multirow{2}{*}{$\begin{array}{c}\text { Total } \\
\text { samples }\end{array}$} \\
\cline { 2 - 3 } & Positive & Negative & 270 \\
\hline $\begin{array}{c}\text { Rappaport Vassiliadis } \\
\text { broth (RV) }\end{array}$ & 134 & 136 & 270 \\
\hline Selenite F (SF) & 109 & 161 & 270 \\
\hline Total* & 147 & 123 & 270 \\
\hline
\end{tabular}

*The overall culture results regardless of the broth type.

Table 2: Distribution of positive samples according to the specimen type in RV and SF broths.

\begin{tabular}{|c|c|c|c|c|c|}
\hline \multirow{2}{*}{\multicolumn{2}{|c|}{$\begin{array}{c}\text { Enrichment broth } \\
\text { Cloacal Swab }\end{array}$}} & \multicolumn{3}{|c|}{ Specimen type } & \multirow{3}{*}{\begin{tabular}{|c|} 
Total \\
134
\end{tabular}} \\
\hline & & \multirow{2}{*}{$\begin{array}{c}\text { Water* } \\
89\end{array}$} & \multirow{2}{*}{$\begin{array}{c}\text { Feces } \\
29\end{array}$} & \multirow[b]{2}{*}{16} & \\
\hline RV & Positive Count & & & & \\
\hline & $\begin{array}{c}\% \text { within } \\
\text { Specimen type }\end{array}$ & $52.0 \%$ & $36.7 \%$ & $80.0 \%$ & $49.6 \%$ \\
\hline \multirow[t]{2}{*}{ SF } & Positive Count & 72 & 21 & 16 & 109 \\
\hline & $\begin{array}{c}\% \text { within } \\
\text { Specimen type }\end{array}$ & $42.1 \%$ & $26.6 \%$ & $80.0 \%$ & $40.4 \%$ \\
\hline \multirow[t]{2}{*}{ Total } & Count & 171 & 79 & 20 & 270 \\
\hline & $\begin{array}{c}\% \text { within } \\
\text { Specimen type }\end{array}$ & $100.0 \%$ & $100.0 \%$ & $100.0 \%$ & $100.0 \%$ \\
\hline
\end{tabular}

*Terrarium water is the water in which the reptiles defecate in. 


\section{Statistical analysis}

The Chi-square test revealed that there was a strong difference between the proportion of positive Salmonella culture results in the two enrichment broths used in favor of the broth RV ( $p$ value $=0.031$ ). In addition, the estimated difference between the positive proportion of RV and SF was 0.092 With a 95\% confidence interval $(0.009,0.176)$.

In addition, according to the Chi-square test the positive proportions of various types of specimens were found significantly different in each broth ( $\mathrm{p}$ value $=0.001$ and 0.000 in RV and SF, respectively). Using multiple comparison with Bonferroni correction showed that terrarium water samples were less likely to be positive than feces or cloacal swabs, while feces were the most likely to give positive culture results in both enrichment broths (Table 2).

Results of our study showed that more than half of the reptiles shed Salmonella in their feces (54.4\%), with the use of two enrichment broths (Rappaport Vassiliadis and Selenite F) and three sample types (feces, cloacal swabs and terrarium water). Healthy reptiles have been shown to excrete Salmonella in their feces according to studies [12] and keeping reptile pets could be considered a menace to people who are immuno-compromised [13]; therefore, the considerable shedding rate in the reptiles of our study does not seem uncommon. Rappaport Vassiliadis broth was found superior to Selenite F for enrichment of reptile feces and cloacal swab samples for Salmonella isolation in our study $(\mathrm{p}=0.031)$. This finding agrees with other researchers who reported the superiority of Rappaport Vassiliadis broth to other enrichment broths for recovery of Salmonella from poultry [14], meat products [15] as well as seawater that contains low or moderate pollution levels [16].

Our results also showed that the type of specimen used to detect Salmonella can possibly affect the positivity of the results in both tested enrichment broths; in that feces samples were more likely to be positive than other samples while terrarium water samples were less likely to be positive for salmonella. Feces contain more of the microorganisms shed by the intestines compared to cloacal swabs and the terrarium water (in which the reptile defecates in); thus, making our finding somehow reasonable. This finding also agrees with other researchers since a considerable difference in the positivity of cloacal swab samples obtained from a laying hen farm was reported compared to the feces samples [17]. Another study in layer breeder flocks also found similar results reporting
$21.4 \%$ Salmonella positive rate in the wet feces compared to $8 \%$ in cloacal swabs [18]. Having said that, we should also mention that the uneven and variant distribution of our samples among the three groups of specimen type could have impacted the results and larger scale studies are warranted.

\section{Conclusion}

Our study showed that Rappaport Vassiliadis broth is a more reliable enrichment broth than Selenite F for detection of Salmonella in reptile feces and cloacal swabs. We also recommend that in case of assessment of intestinal shedding, feces samples be used whenever possible for Salmonella recovery in reptiles or other animals. The considerable rate of Salmonella shedding in the healthy reptiles' feces which was found in our study also warrants cautious care in the handling of young children or immunocompromised people to all reptilian species.

\section{Acknowledgement}

Authors would like to thank Dr Alireza Bahonar and Dr Ashrafi for their kind help throughout this project.

\section{Conflict of Interest}

The authors have no conflict of interest to declare.

\section{Bibliography}

1. Selbitz HJ., et al. "Das Salmonellen-problem. Salmonellen als Erreger von Tierseuchen und Zoonosen". Gustav Fischer Verlag, Jena-Stuttgart (1995): 18-51.

2. Turnbull PCB. "Food poisoning with special reference to Salmonella - its epidemiology, pathogenesis and control". Clinical Gastroenterology 8 (1979): 586-594.

3. Woodward DL., et al. "Human salmonellosis associated with exotic pets”. Journal of Clinical Microbiology 35 (1997): 27862790.

4. Chiodini R J and Sundberg J P. "Salmonellosis in reptiles: a review". American Journal of Epidemiology 113 (1981): 494.

5. Milstone AM., et al. "Alerting pregnant women to the risk of reptile-associated salmonellosis". Obstetrics and Gynecology 107 (2006): 516-518. 
6. Mermin J., et al. "Iguana and Salmonella Marina infection in children: A reflection of the increasing incidence of reptileassociated Salmonellosis in the United States". Pediatrics 99 (1997): 399-402.

7. Cohen ML., et al. "Turtle-associated salmonellosis in the United States - effect of public health action, 1970-1976". JAMA 243 (1980): 1247-1249.

8. Wegener HC., et al. "Pork as a source of human salmonellosis". II International Symposium on Epidemiology and Control of Salmonella in Pork, Copenhagen (1997): 3-8.

9. Love B C and Rostagno M H. "Comparison of Five Culture Methods for Salmonella Isolation from Swine Fecal Samples of Known Infection Status". Journal of Veterinary Diagnostic Investigation 20.5 (2008): 620-624.

10. Michael GB., et al. "Comparison of different selective enrichment steps to isolate Salmonella sp from feces of finishing swine”. Brazilian Journal of Microbiology 34 (2003): 138-142.

11. Harvey RW and Price TH. "Salmonella isolation from reptilian faeces: a discussion of appropriate cultural techniques". Journal of Hygiene (Lond) 91.1 (1983): 25-32.

12. Pasmans F., et al. "Characterization of Salmonella isolates from captive lizards". Veterinary Microbiology 110 (2005): 285-291.

13. Centers for Disease Control and Prevention (CDC). "Multistate outbreak of human Salmonella typhimurium infections associated with pet turtle exposure - United States, 2008". Morbidity and Mortality Weekly Report 59 (2010): 191-196.

14. Rall VLM., et al. "Evaluation of three enrichment broths and five plating medium for Salmonella detection in poultry". Brazilian Journal of Microbiology 36.2 (2005): 147-150.

15. Kalapothaki V., et al. "Comparison of Rappaport-Vassiliadis Enrichment Medium and Tetrathionate Brilliant Green Broth for Isolation of Salmonellae from Meat Products". Journal of Food Protection 46.7 (1983): 618-621.

16. Morin`igo MA., et al. "Laboratory study of several enrichment broths for the detection of Salmonella spp. particularly in relation to water samples". Journal of Applied Bacteriology 74 (1993): 330-335.
17. García C., et al. "Assessment of Salmonella spp. in feces, cloacal swabs, and eggs (eggshell and content separately) from a laying hen farm". Poultry Science 90.7 (2011): 1581-1585.

18. Serpil KAHYA., et al. "Comparison of Different Sample Types for Salmonella Detection From Chicken Layer Breeder Flocks". Uludağ Üniversitesi Veteriner Fakültesi Dergisi 32.2 (2013): 1924.

\section{Volume 3 Issue 9 September 2021} (C) All rights are reserved by Naqa Saleh Mahdi Tamimi., et al. 